\title{
RTDS-based Model Component Development of a Tri-axial HTS Power Cable and Transient Characteristic Analysis
}

\author{
Sun-Kyoung Ha*, Sung-Kyu Kim*, Jin-Geun Kim*, Minwon Park*, In-Keun Yu ${ }^{\dagger}$, \\ Sangjin Lee ${ }^{* *}$, Jae-Ho Kim*** and Kideok Sim ${ }^{\S}$
}

\begin{abstract}
The transient characteristics of the tri-axial High Temperature Superconducting (HTS) power cable are different from those of a conventional power cable depending on whether the cable is under a steady or transient state due to the quench. Verification using simulation tools is required to confirm both the characteristics of the cable and the effect of the cable when it is applied to a real utility. However, a component for the cable has not been provided in simulation tools; thus the RTDSbased model component of the tri-axial HTS power cable was developed, and a simulation was performed under the transient state. The considered properties of model component include resistance, reactance and temperature. Simulation results indicate the variation of HTS power cable condition. The results are used for the transient characteristic analysis and stability verification of the tri-axial HTS power cable. In the future, the RTDS-based model component of the cable will be used to implement the hardware-in-the-loop simulation with a protection device.
\end{abstract}

Keywords: Circuit simulation, HTS, HTS power cable, RTDS, Tri-axial cable

\section{Introduction}

High Temperature Superconducting (HTS) power cables are compact and have high current density. So these cables are expected to effectively solve the problems caused by increases in electricity demand and limited space. A triaxial HTS power cable with a mutually layered structure of three phases has advantages, including a reduced amount of HTS wires and a small cryogenic surface area compared with other HTS power cables. One tri-axial HTS power cable can take the place of 18 large conventional copper cables operating at the same voltage level $[1,2]$. Before using the tri-axial HTS power cable in a commercial installation, the system should be analyzed using simulation tools in order to confirm both the characteristics and the effects of the cable. The transient characteristics of the triaxial HTS power cable are different from those of other HTS power cables as well as conventional copper power cables depending on the structure and whether the cable is under a steady or transient state due to the quench [3].

In this paper, for the simulation analysis, a tri-axial HTS power cable model component was developed for a RealTime Digital Simulator (RTDS). The considered properties

$\dagger$ Corresponding Author: Dept. of Electrical Engineering, Changwon National University, Changwon, Korea. (yuik@cwnu.ac.kr)

* Dept. of Electrical Engineering, Changwon National University, Changwon, Korea. (yuik@cwnu.ac.kr)

** Division of Energy \& Electrical Engineering, Uiduk University, Gyeongju, Korea. (sjlee@uu.ac.kr)

*** Dept. of Fire and disaster prevention, Daejeon University, Daejeon, Korea. (jkim@dju.ac.kr)

$\S \quad$ Korea Electrotechnology Research Institute, Changwon, Korea (skedy0509@keri.re.kr)

Received: August 29, 2013; Accepted: May 12, 2015 of the model component include the resistance, reactance, and temperature variations of the HTS wires and Liquid Nitrogen $\left(\mathrm{LN}_{2}\right)$. The cable resistance was obtained based on a characteristic equation of superconductor [4], and it was also changed by the operating current, critical current, and temperature of the cable. The reactance was determined based on its structure and properties, such as permittivity. The temperature variations were calculated considering the heat value and heat transfer. The model component was applied to a model power system, and a simulation was performed under a transient state using the RTDS. The simulation results showed the effect of the tri-axial HTS power cable on the power system, such as the fault current of the three phases, and the characteristic variations in the tri-axial HTS power cable, such as quench resistance and the surface temperature of the HTS wires under fault conditions. The simulation results are presented and discussed in detail. The approach presented in the RTDSbased model component of the cable will be used to implement the hardware-in-the-loop simulation with a protection device. Also, this work can be used for other types of HTS power cables to investigate their transient characteristics.

\section{RTDS-based Model Component Development}

\subsection{Tri-axial HTS power cable}

The tri-axial HTS power cable, which has a multi-layer structure, contains a core, three-conducting layers, and a shield layer in a cryostat. The conducting layers are twisted 


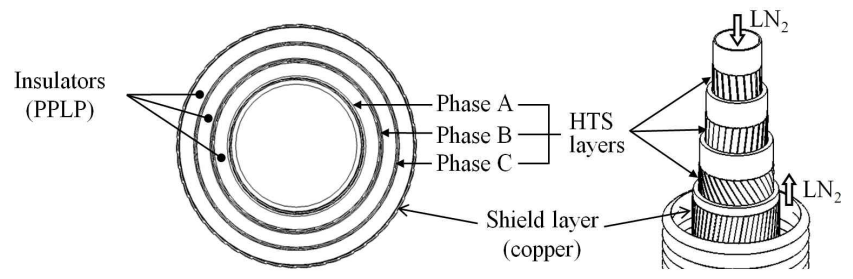

Fig. 1. Structure of the tri-axial HTS power cable

clockwise and counter-clockwise along the same axis. The conducting layers are insulated using cryogenic dielectric material, which comprises PolyPropylene Laminated Paper (PPLP). In order to obtain the superconducting state of the cable, it is refrigerated by $\mathrm{LN}_{2}$, which is injected into the inner core pipe and refluxed through the outer cryostat [5]. Fig. 1 describes the structure of the tri-axial HTS power cable. This structure has the advantages of effectiveness and economic feasibility, but it also has disadvantages in terms of the insulation design and the imbalance of the three-phase currents. The detailed design processes are referred to in previous papers $[6,7]$. Table 1 indicates the design parameters of the cable.

Table 2 shows the parameter-dimensions of the tri-axial HTS power cable [8]. In Table 2, the flow rate, the $\mathrm{LN}_{2}$ temperature of the input terminal, and the pressure of the input terminal are obtained by considering the relation formulas of the pressure loss, flow rate, and the $\mathrm{LN}_{2}$ temperature variations along with the length [9].

The parameters given in Table 2 are required to calculate $\mathrm{LN}_{2}$ temperature and the $\mathrm{LN}_{2}$ temperature is used to calculate the HTS wire surface temperature of three phases and then the surface temperature is used for the cable resistance calculation. These parameters were applied to the developed model component in the RSCAD/RTDS.

Table 1. Parameters of the tri-axial HTS power cable

\begin{tabular}{l|c}
\hline \multicolumn{1}{c|}{ Parameters } & Values \\
\hline Voltage/Capacity & $22.9 \mathrm{kV} / 50 \mathrm{MVA}$ \\
\hline Rated current & $1,260 \mathrm{~A}_{\text {rms }}$ \\
\hline DC critical current per wire & $110 / 100 / 100 \mathrm{~A} @ 77 \mathrm{~K}$, self-field \\
\hline HTS wire & YBCO (width: $4 \mathrm{~mm})$ \\
\hline Number of HTS wires & $25 / 31 / 30$ \\
\hline Insulation thickness & $4 \mathrm{~mm}(\mathrm{PPLP})$ \\
\hline Radius of each layer & $17 / 21 / 25 / 29 \mathrm{~mm}$ \\
\hline $\begin{array}{l}\text { Pitch length and winding direction } \\
\text { of each layer }\end{array}$ & $+998 /-996 /+216 /-986 \mathrm{~mm}$ \\
\hline Cable length & $1 \mathrm{~km}$ \\
\hline
\end{tabular}

Table 2. Parameter-dimensions of the tri-axial HTS power cable

\begin{tabular}{l|c}
\hline \multicolumn{1}{c|}{ Parameters } & Values \\
\hline Electricity heat value/External intrusion heat value & $3 / 2.5 \mathrm{~W} / \mathrm{m}$ \\
\hline $\mathrm{LN}_{2}$ temperature of input terminal & $66 \mathrm{~K}$ \\
\hline Flow rate of $\mathrm{LN}_{2}$ & $0.3 \mathrm{~kg} / \mathrm{s}$ \\
\hline Pressure of input terminal & $1 \mathrm{MPa}$ \\
\hline Cooling capacity of hybrid cooling system & $5.8 \mathrm{~kW} @ 66.4 \mathrm{~K}$ \\
& $8 \mathrm{~kW} @ 77 \mathrm{~K}$ \\
\hline
\end{tabular}

\subsection{Modeling process of the model component in RTDS}

RTDS is a fully digital electromagnetic transient power system simulator that operates in real time. Furthermore, the RTDS provides fast, reliable, accurate, and cost-effective results for power systems with complex high-voltage alternating currents as well as high-voltage direct current networks [10]. It can also be used to implement hardware in a loop simulation because it operates in real time.

The principal output results of the model component for a tri-axial HTS power cable are the resistance and reactance. The reactance is simply obtained using an equation based on the cable shape [6]. However, the resistance should be calculated considering the operating temperature, current and critical current because it changes according to both the cable shape and cable state. In addition, these results provide the temperature variations of the HTS wire and $\mathrm{LN}_{2}$, and the required cooling capacity after the transient state.

Thus the inductance and capacitance were calculated using the following Eqs. [11]. $L_{\text {self }}$ is the self-inductance (1), $M_{i j}$ is the mutual inductance (2) and $C_{i j}$ is the capacitance (3).

$$
\begin{gathered}
L_{\text {self }}=\frac{\pi \mu_{0} r_{i}^{2}}{L_{p i}{ }^{2}}+\frac{\mu_{0}}{2 \pi} \ln \frac{D}{r_{i}}[\mathrm{H} / \mathrm{m}] \\
M_{i j}=\frac{a_{i} a_{j} \pi \mu_{0} r_{i}^{2}}{L_{p i} L_{p j}}+\frac{\mu_{0}}{2 \pi} \ln \frac{D}{r_{j}}[\mathrm{H} / \mathrm{m}] \\
C_{i j}=\frac{2 \pi \varepsilon_{0} \varepsilon_{r}}{\ln \frac{r_{j}}{r_{i}}}[\mathrm{~F} / \mathrm{m}]
\end{gathered}
$$

where $r_{i}$ is the radius of the inner layer, $r_{j}$ is the radius of the outer layer, $\mu_{0}$ is the permeability of the free space, $l_{p i}$ and $l_{p j}$ are the winding pitches of each layer, $a_{i}$ and $a_{j}$ are the 1(clockwise) and the -1 (counter-clockwise) winding directional factors of the i-layer and the j-layer, respectively. $D$ is the current return path, $\varepsilon_{0}$ is the permittivity of the free space, and $\varepsilon_{r}$ is the relative permittivity of the dielectric material which is PPLP. Its permittivity is 2.2 [6].

The resistance of the cable was calculated based on a characteristic equation of superconductor (4). The equation was obtained by experiment and it requires the critical current, operating current, and temperature [4]. Also this resistance was calculated considering the parallel connection with a base metal.

$$
\begin{aligned}
R=e^{\left(\frac{\mathrm{I}}{\left.\left(-1.20531+0.20913 *\left(\mathrm{Ic}-\left(\frac{\mathrm{Ic}}{50} *(\mathrm{~K}-77)\right)-0.27 * \frac{\mathrm{I}}{2}\right)\right)\right)}\right)} \\
* \frac{\left.2.14242 \cdot 10^{-6}+1.43695 \cdot 10^{-4}\right)}{I}
\end{aligned}
$$


where $R$ is the cable resistance, $I c$ is the critical current, $K$ is the temperature, and $I$ is the operating current.

The temperature was calculated using the heat value Eq. (5) and the heat transfer Eq. (6) [11]. The calculation of the $\mathrm{LN}_{2}$ temperature was divided into two parts, i.e., the injected $\mathrm{LN}_{2}$ and refluxed $\mathrm{LN}_{2}$, and it was assumed to operate under the peak temperature value along with the length of each path and the radial axis transfer.

$\mathrm{LN}_{2}$ temperature of input terminal is $66 \mathrm{~K}$ as shown in Table 2, and $\mathrm{LN}_{2}$ peak temperature values along with the length of inner pipe and outer pipe are $75.3 \mathrm{~K}$ and $76.2 \mathrm{~K}$, respectively. These were calculated based on a reference [9].

The authors assumed that all electrical power loss was converted into thermal energy because the cable didn't have any mechanical loss. The generated heat value was calculated using the current and the resistance of the conductor as in (5).

$$
Q_{\text {gen }}=I^{2} R t \quad[\mathrm{~J}]
$$

where $I$ is the conductor current, $R$ is the conductor resistance, $t$ is the time step.

The heat transfer rate of the cable layers was calculated using the heat conduction equation of a hollow cylinder as in (6) [12].

$$
Q_{\text {cond }}=\frac{2 \pi L k\left(T_{1}-T_{2}\right)}{\ln \frac{r_{2}}{r_{1}}}
$$

where $L$ is the length of the cable, $\left(T_{1}-T_{2}\right)$ is the temperature difference between the cable layers, $r_{l}$ is the radius of the inner layer, $r_{2}$ is the radius of the outer layer and $k$ is the thermal conductivity of the materials. The thermal conductivity of PPLP is $0.2 \mathrm{~W} / \mathrm{mK}$. The heat transfer rate of in-between phases (a, b, and c) and shield layers of the cable shown in Fig. 1 were calculated in every time step using the parameters given in Table 3.

Fig. 2 illustrates overall procedure of the calculation algorithm for the surface temperature of the cable layers in

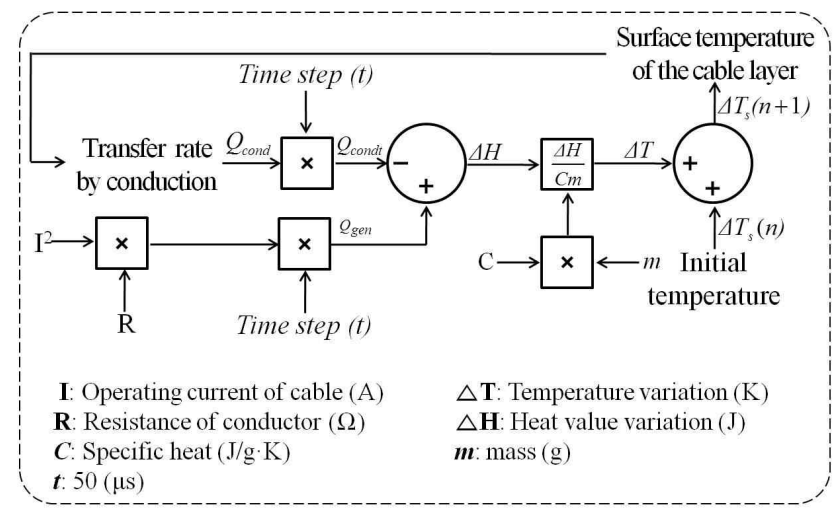

Fig. 2. Calculation algorithm of the surface temperature
Table 3. The parameters for the surface temperature calculations

\begin{tabular}{c|c|c|c|c}
\hline \multirow{2}{*}{ Layers } & \multicolumn{4}{|c}{ Parameters } \\
\cline { 2 - 5 } & $\mathrm{T}_{1}$ & $\mathrm{~T}_{2}$ & $\mathrm{r}_{1}$ & $\mathrm{r}_{2}$ \\
\hline \multirow{2}{*}{ Phase A } & Phase A & $\mathrm{LN}_{2}$ & $\mathrm{LN}_{2}$ & Phase A \\
\cline { 2 - 5 } & Phase A & Phase B & Phase A & Phase B \\
\hline \multirow{2}{*}{ Phase B } & Phase B & Phase A & Phase A & Phase B \\
\cline { 2 - 5 } & Phase B & Phase C & Phase B & Phase C \\
\hline \multirow{2}{*}{ Phase C } & Phase C & Phase B & Phase B & Phase C \\
\cline { 2 - 5 } & Phase C & Shield & Phase C & Shield \\
\hline \multirow{2}{*}{ Shield } & Shield & Phase C & Phase C & Shield \\
\cline { 2 - 5 } & Shield & $\mathrm{LN}_{2}$ & Shield & $\mathrm{LN}_{2}$ \\
\hline
\end{tabular}

detail. As shown in Fig. 2, the heat value $Q_{g e n}$ and the transfer value $Q_{\text {cond }}$ are accumulated every time step ( $\Delta H=$ $\left.Q_{\text {gen }}+Q_{\text {condt }}\right)$ in order to evaluate the temperature variation value $\Delta T$ as in (7).

$$
\Delta T=\frac{\Delta H}{m C}
$$

where $m$ is the mass, $C$ is the specific heat. Finally the surface temperature of the cable layer $T_{s}$ will be integrated for the required time duration as in (8).

$$
\mathrm{T}_{\mathrm{s}}(\mathrm{n}+1)=\mathrm{T}_{\mathrm{s}}(\mathrm{n})+\Delta \mathrm{T}
$$

where $n$ is the iteration number.

Shield layer resistance was calculated using the specific resistance Eq. (9) and it was also changed by the temperature.

$$
C_{c u}=(-0.246499+0.06351 T) \cdot 10^{-9} \quad[\Omega \cdot \mathrm{m}]
$$

where $C_{c u}$ is the specific resistance, $T$ is the temperature.

\section{Application of the Model Component}

\subsection{Configuration of a $22.9 \mathrm{kV} / 50 \mathrm{MVA}$ class power system}

The developed model component was applied to a 22.9 $\mathrm{kV}$ class power system model, which consisted of a $5 \mathrm{~km}$ distribution line made of ACSR (aluminum conductor steel reinforced) 160 and a 50 MVA load [3]. The impedance of the distribution line was $0.506+\mathrm{j} 0.0025 \Omega$. The fault logic was in front of the load. This power system's circuit diagram is shown in Fig. 3. The developed component took the operating current and gave the variable inductance, capacitance, and resistance in the power system circuit.

\subsection{Transient state characteristic analysis}

The simulation was performed under a transient state to analyze both the characteristics of the cable and the effect 


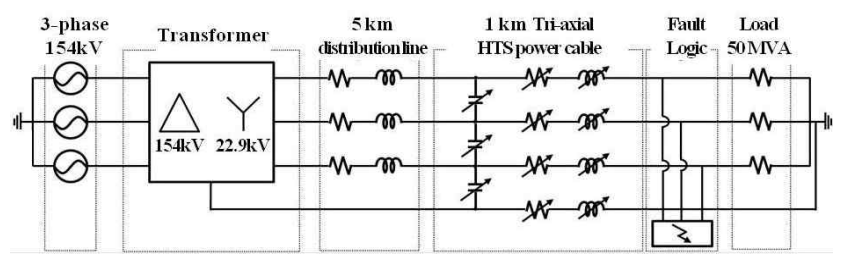

Fig. 3. Configuration of a $22.9 \mathrm{kV} / 50 \mathrm{MVA}$ class power system in the RTDS

of the cable when it is applied to a real utility. The simulation was implemented on a three-phase fault. The rated current was $1.26 \mathrm{kA}_{\mathrm{rms}}$, and the $\mathrm{DC}$ critical currents of each layer were $2.75 \mathrm{kA}, 3.1 \mathrm{kA}$, and $3 \mathrm{kA}$ at $77 \mathrm{~K}$, respectively. The fault resistance was $1.59 \Omega$. The threephase fault was initiated at $0.2 \mathrm{~s}$ and lasted for $0.066 \mathrm{~s}$.

Fig. 4 and Fig. 5 indicate the simulation results of the three-phase fault according to the electrical and thermal characteristics of the cable. Fig. 4(a) and (b) show the current and cable resistance, indicating the root mean square value. Fig. 5(a) and (b) show the temperatures of the surface of each phase and the $\mathrm{LN}_{2}$. In these results, variations in the surface temperature and resistance are important.

As soon as a fault was initiated, the fault current reached $9.2 \mathrm{kA}_{\mathrm{rms}}$, and as a result, the resistance increases due to the quench and the peak temperature of the $\mathrm{LN}_{2}$ slight increased by $0.17 \mathrm{~K}$. Although the DC critical current of phase $\mathrm{B}$ was higher than that of other phases as given in Table 1, the quench resistance of phase B was not small, as depicted Fig. 4 (b). This phenomenon was caused by the

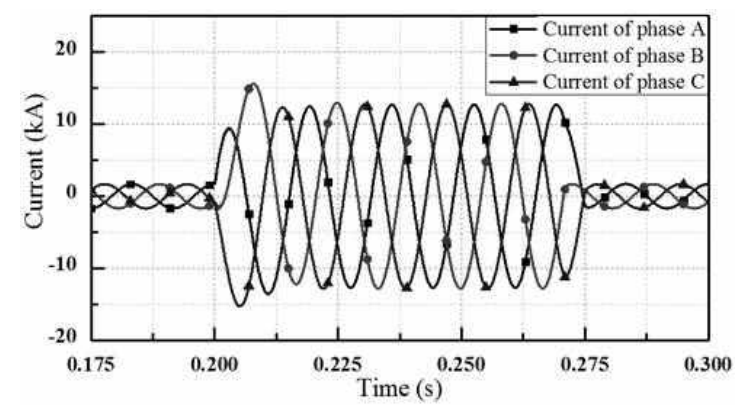

(a)

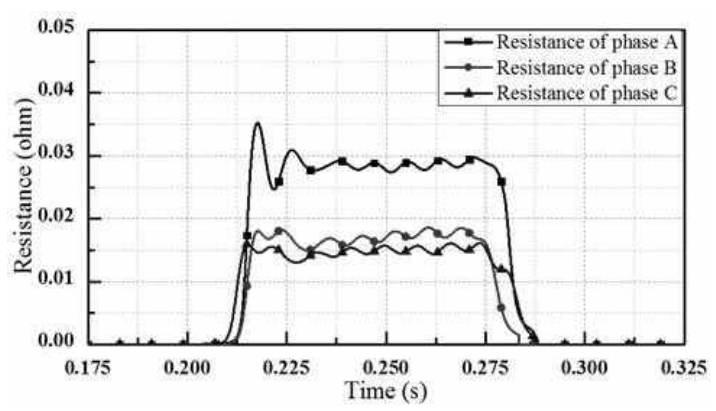

(b)

Fig. 4. Simulation results of the three-phase fault; (a) Fault current; (b) Resistance of the HTS power cable under transient condition

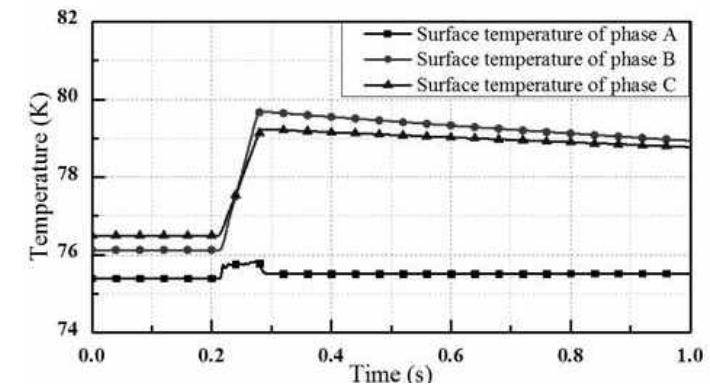

(a)

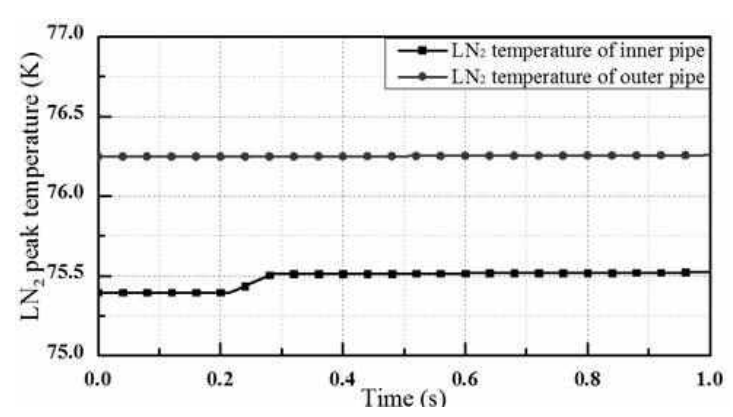

(b)

Fig. 5. Thermal characteristics of the tri-axial HTS power cable under the three-phase fault; (a) Surface temperatures of each phase; (b) Peak temperature of $\mathrm{LN}_{2}$

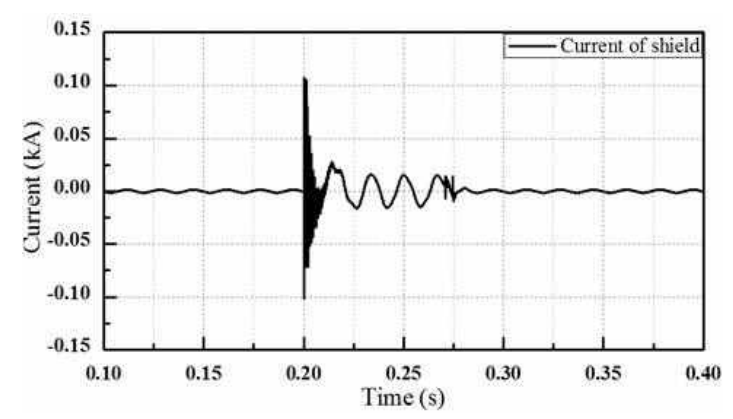

Fig. 6. Shield layer current of the HTS power cable under transient condition

decrease of critical current of phase B. The decrease was caused by the degradation of the cooling system by the structural characteristic of the cable. If the surface temperatures increase higher than the boiling point of $\mathrm{LN}_{2}$, bubbles would have influence on the electrical insulation properties. This process is prevented by the cooling system control and the additional stabilizer that can bypass the fault current.

Fig. 6 shows the shield layer current. The shield layer also has resistance and reactance. The resistance is $0.441 \Omega$ at $77 \mathrm{~K}$ and the cooling characteristic is the same as phase A layer because the layer is in direct contact with the refrigerant. Unbalanced currents flow through the shield layer, and the unbalance current is caused by not only inherent imbalance and the different critical currents of each phase but also the unbalance faults. In this case, 
although the unbalance current occurred, the current and resistance were small, so the shield layer did not affect the other phase.

\section{Conclusion}

This paper illustrated the structure and transient characteristics of a tri-axial HTS power cable. The RTDSbased model component of the cable was developed to investigate the transient characteristics of the cable, and it was applied to a power system model. The transient simulation was performed on a three-phase fault. The simulation results of the three-phase fault with the tri-axial HTS power cable represent the fault current, cable resistance, surface temperature of each phase, and peak temperature of $\mathrm{LN}_{2}$. These results indicated that the tri-axial HTS power cable could suffer catastrophic loss of superconductivity (quench) during a major transient event.

As a result, by developing the model component, a realtime simulation of the power system with the tri-axial HTS power cable was possible. The RTDS-based model component of the cable will be used to implement the hardware-in-the-loop simulation with a protection device. This work will also effectively be used to develop model components of other types of HTS power cables and to analyze their transient characteristics in connection with real power systems.

\section{Acknowledgements}

This research was supported by Basic Science Research Program through the National Research Foundation of Korea (NRF) funded by the Ministry of Education (NRF2013R1A1A1010676)

\section{References}

[1] D. Lindsay, M. Roden, R. Denmon, D. Willen, B. Mehraban, and A. Keri, "Installation and commissioning of triax hts cable," JICABLE, A3-2, 2007.

[2] Website: http://www.supercables.com/

[3] J. H. Bang, H. H. Je, J. H. Kim, K. Sim, J. Cho, J. Y. Yoon, M. Park, and I. K. Yu, "Critical Current, Critical Temperature and Magnetic Field Based EMTDC Model Component for HTS Power Cable," IEEE Trans. Appl. Supercond., Vol. 17, pp. 1726-1729, 2007.

[4] J. H. Bang, M. Park, and I. K. Yu, "HTS power cable component for PSCAD/EMTDC," Patent Publication, 10-2007-1016246, 2007.

[5] S. K. Ha, S. K. Kim, J. G. Kim, M. Park, I. K. Yu, S. Lee, K. Sim, and A. R. Kim, "Transient Characteristic Analysis of a Tri-axial HTS Power Cable using PSCAD/EMTDC." IEEE Trans. Appl. Super- cond., to be published.

[6] S. K. Ha, S. K. Kim, J. G. Kim, M. Park, I. K. Yu, S. Lee, and K. Sim, "Development of an impedance matching program for balancing the distribution in a tri-axial HTS power cable," The International Conference on Superconductivity and Magnetism, to be published.

[7] S. K. Kim, S. K. Ha, J. G. Kim, S. Kim, M. Park, I. K. Yu, S. Lee, K. Sim, "Design and AC loss analysis of a 22.9 kV/50 MVA class tri-axial HTS power cable ," The International Conference on Superconductivity and Magnetism, to be published.

[8] H. S. Yang, D. L. Kim, S. H. Sohn, J. H. Lim, Y. S. Choi, and S. D. Hwang, "Hybrid Cooling System Installation for the KEPCO HTS Power Cable," IEEE Trans. Appl. Supercond., vol. 20, no. 3, pp. 1292-1295, 2010.

[9] C. H. Lee, D. H. Kim, C. D. Kim, K. S. Kim, and I. S. Kim, "An optimal cooling method for long HTS power transmission cable," The Korea Institute of Applied Superconductivity and Cryogenics, vol. 6, no. 3, pp. 56-61, 2004.

[10] G. H. Kim, M. Park, and I. K. Yu, "RTDS-based real time simulations of grid-connected wind turbine generator systems," in APEC2010, Feb. 2010, pp. 2085-2090.

[11] J. H. Lee, G. Cha, "AC loss calculation of a multilayer HTS transmission cable considering the twist of each layer," IEEE Trans. Appl. Supercond. 1, 24332436, 2001.

[12] Incropera, Dewitt, Bergman, Lavine White, Introduction to heat transfer, fifth ed., John wiley \& Sons, New York, 2007.

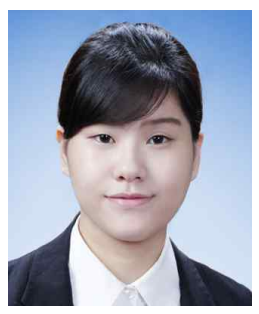

Sun-Kyoung Ha She received B.S degree in electrical engineering from Uiduk University. Her research interests are design and transient analysis of the HTS power cable.

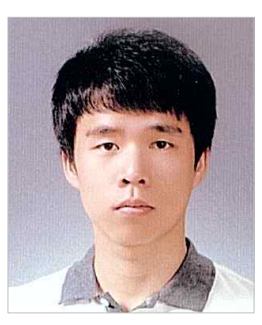

Sung-Kyu Kim He received B.S. and M.S. degrees in Electrical Engineering from Changwon National University, Changwon, Korea, in 2008 and 2012, respectively. $\mathrm{He}$ is currently working toward Ph.D. degree in Electrical Engineering at Changwon National University, Changwon, Korea. His fields of interest are high voltage direct current system and superconducting power cable. 


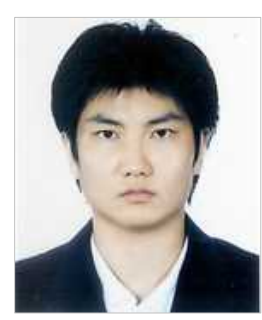

Jin-Geun Kim He received B.S, M.S. and Ph.D. degrees in Electrical Engineering from Changwon National University in 2008, 2010 and 2014, respectively. His research interests are superconducting cable and HVDC transmission system.

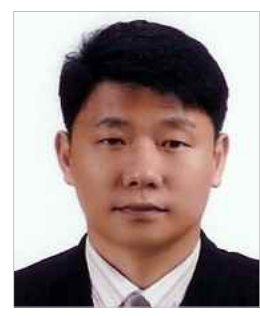

Minwon Park He received B.S degree in Electrical Engineering from Changwon National University in 1997 and his M.S. and Ph.D. degrees in Electrical Engineering from Osaka University in 2000 and 2002, respectively. His research interests are development of the simulation model of power conversion equipment and renewable energy sources using EMTP type Simulators.

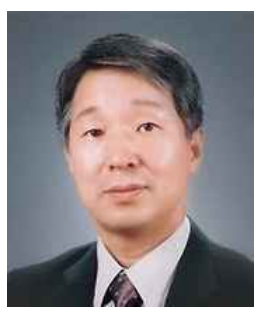

In-Keun Yu He received B.S degree in Electrical Engineering from Dongguk University in 1981 and his M.S. and Ph.D. degrees in Electrical Engineering from Hanyang University in 1983 and 1986, respectively. His research interests are electric energy storage and control systems, PSCAD/EMTDC and RTDS simulation studies, and renewable energy sources.

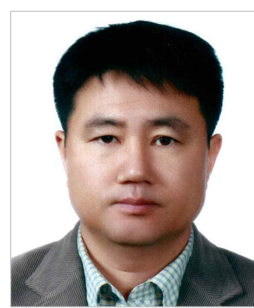

Sangjin Lee He received B.S, M.S., and Ph.D. degrees in Electrical Engineering from Yonsei University in 1995, 1991, and 1988, respectively. His research interests are development of superconducting fault current limiter for power system.

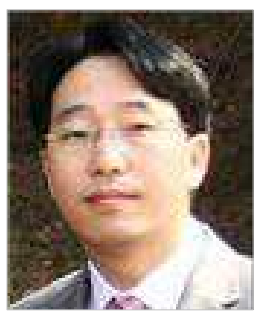

Jae-Ho Kim He received Ph.D. degree in Electrical Engineering from Changwon National University in 2010 and he has worked as a post-doctor in Center for Advanced Power Systems (CAPS) at Florida State University from 2009 2012. His research interests are superconducting cable and its

protection system.

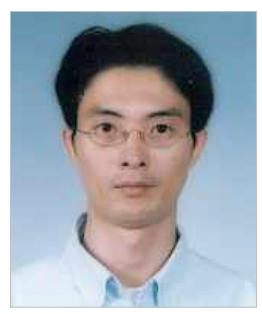

Kideok Sim He received B.S, M.S. and $\mathrm{Ph} . \mathrm{D}$. degrees in Electrical Engineering from Yonsei University in 1997, 1999, and 2011, respectively. His research interests are the design and analysis of transport characteristics of HTS power cable. 